\title{
Omnidirectional transport and navigation of Janus particles through a nematic liquid crystal film
}

\author{
Dinesh Kumar Sahu, ${ }^{1}$ Swapnil Kole, ${ }^{2}$ Sriram Ramaswamy $\odot,{ }^{2}$ and Surajit Dhara $\odot^{1, *}$ \\ ${ }^{1}$ School of Physics, University of Hyderabad, Hyderabad 500 046, India \\ ${ }^{2}$ Centre for Condensed Matter Theory, Department of Physics, Indian Institute of Science, Bangalore 560 012, India
}

(Received 29 November 2019; revised 20 February 2020; accepted 17 June 2020; published 8 July 2020)

\begin{abstract}
We create controllable active particles in the form of metal-dielectric Janus colloids which acquire motility through a nematic liquid crystal film by transducing the energy of an imposed perpendicular AC electric field. We achieve complete command over trajectories by varying the field amplitude and frequency, piloting the colloids at will in the plane spanned by the axes of the particle and the nematic. The underlying mechanism exploits the sensitivity of electro-osmotic flow to the asymmetries of the particle surface and the liquid crystal defect structure. We present a calculation of the dipolar force density produced by the interplay of the electric field with director anchoring and the contrasting electrostatic boundary conditions on the two hemispheres, which accounts for the dielectric-forward (metal-forward) motion of the colloids due to induced puller (pusher) force dipoles. These findings open unexplored directions for the use of colloids and liquid crystals in controlled transport, assembly, and collective dynamics.
\end{abstract}

DOI: 10.1103/PhysRevResearch.2.032009

Electrophoresis, the use of electric fields to transport tiny particles through fluids, is an important technology for macromolecular sorting, colloidal assembly, and display devices and a challenging area of soft-matter research [1-8]. Classic electrophoresis is linear: ions in the electrical double layer drag the fluid, and hence the particle itself, with a velocity proportional to and parallel to the applied field. Inducedcharge electro-osmosis of particles is nonlinear: the applied field itself creates the double layer. Polarity in the shape or surface properties of the particle results in a flow pattern that picks out a direction of motion, with a velocity quadratic in and normal to the field [9-13]. Neither effect offers the option of continuously tuning the direction of transport and hence the desired motility of the microscopic particles [14,15].

When the ambient fluid is a nematic liquid crystal (NLC), the anchoring of the mean molecular orientation or director $\hat{\mathbf{n}}$ normal to the surface of a suspended homogeneous spherical particle mandates Saturn-ring [16,17] or asymmetric [18,19] defect structures, resulting, respectively, in quadrupolar or dipolar elastic distortions in the NLC [19]. The nonlinear electro-osmotic flow resulting from an imposed electric field yields bidirectional transport of dipolar particles parallel to the local director, thanks to their broken fore-aft symmetry, an effect termed liquid-crystal-enabled electrophoresis [20-24]. The Saturn-ring particles, by contrast, maintain the quadrupolar symmetry of the flow and hence display no motility $[23,24]$.

\footnotetext{
*sdsp@uohyd.ernet.in

Published by the American Physical Society under the terms of the Creative Commons Attribution 4.0 International license. Further distribution of this work must maintain attribution to the author(s) and the published article's title, journal citation, and DOI.
}

Our study focuses on spherical particles with two hemispherical faces: one metal, the other dielectric (see Supplemental Material Fig. S1 [25]). Their "Janus" character is sensed only by the electrostatics of the medium; as far as the mechanics of the ambient NLC is concerned they are elastic quadrupoles. Our central result is that purely by tuning the amplitude and frequency of an imposed electric field, and not its direction, we can achieve guided transport of Janus colloids in the direction of our choosing perpendicular to the field, amounting to the realization of controllable phoretic active particles [26]. Our findings suggest novel possibilities at the interface of colloids and liquid crystals for controlled transport, assembly, nonequilibrium phenomena, and collective dynamics.

We work with a room-temperature NLC mixture, MLC6608. Macroscopic alignment in the $x$ direction is imposed by the treated surfaces of the bounding electrodes parallel to the $x y$ plane [Fig. 1(b)]. Their separation is larger than, but close to, the diameter $2 a$ of the suspended particles [27], which produce a quadrupolar elastic field in the nematic [Fig. 1(a)]. We work in the dilute regime and do not consider cases of higher concentration where aggregation and network formation are important $[28,29]$. Due to the elastic distortion of the director, the particles resist sedimentation and levitate in the bulk [30]. This feature, and therefore liquid-crystalenabled electrophoresis as well, is absent in the isotropic phase. The dielectric anisotropy $\left(\Delta \epsilon=\epsilon_{\|}-\epsilon_{\perp}\right.$, where $\epsilon_{\|}$and $\epsilon_{\perp}$ are the dielectric permittivities for the electric field parallel and perpendicular to $\hat{\mathbf{n}}$ ) of the sample is negative so that the electric field $\mathbf{E}$, applied in the $z$ direction, does not influence the macroscopic director except near the particles [23]. Figure 1(c) shows the optical microscope texture of a Janus quadrupolar particle with cross polarizers. The four-lobed intensity pattern of the particle, a characteristic feature of the elastic quadrupole [27], is further substantiated by the texture obtained by inserting a $\lambda$ plate (inset). The texture without 
(a)
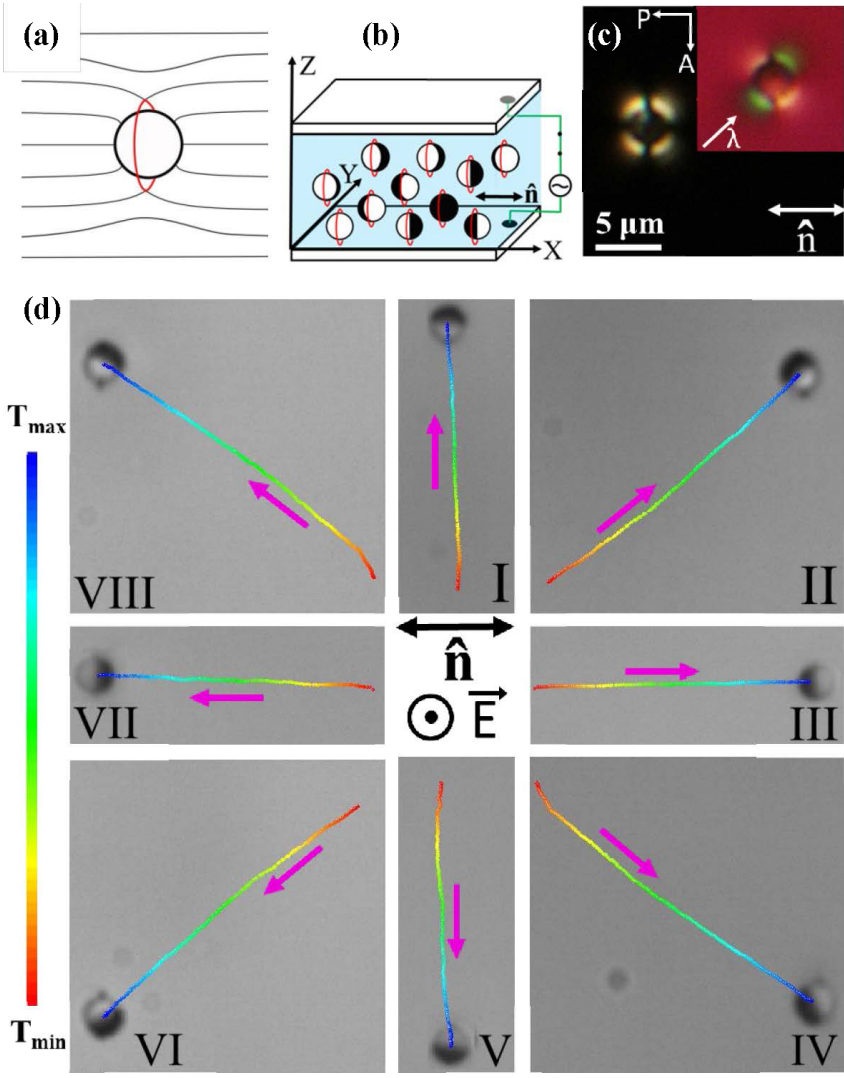

FIG. 1. (a) Quadrupolar distortion of the nematic director around a spherical particle. The red circle represents the Saturn-ring defect. (b) Janus quadrupolar particles in a planar cell with an AC field. The dark hemisphere represents metal. (c) Optical microscope texture of a Janus quadrupolar particle in the NLC between crossed polarizer (P) and analyzer $(\mathrm{A})$ in the $x y$ plane. Inset: Texture with a $\lambda$ plate $(530$ $\mathrm{nm})$. (d) Time-coded trajectories $\left(T_{\min }=0 \mathrm{~s}\right.$ to $T_{\max }=5 \mathrm{~s}$ ), labeled from I to VIII, under an AC electric field $\left(1.54 \mathrm{~V} \mathrm{\mu m}^{-1}, 30 \mathrm{~Hz}\right)$. Trajectories of selected particles are grouped. Pink arrows denote the direction of motion. (See Supplemental Material Movie S1 [25]). The direction of the electric field $(\vec{E})$ and the director $(\hat{\mathbf{n}})$ for all trajectories are shown in the center. Cell thickness: $5.2 \mu \mathrm{m}$.

polarizers shows that the metal hemisphere of particles in the absence of an AC field is oriented in different directions, always keeping the Saturn rings perpendicular to the macroscopic director (see Supplemental Material Fig. S2A [25]). Depending on the anchoring, Janus particles can also induce other types of defects [31,32], which are not considered in this study.

Once the AC electric field is switched on, the particles reorient [14] so that the plane of the metal-dielectric interface lies parallel to the field [Fig. 1(b) and Fig. 1(d)]. With increasing field, they start moving in specific directions in the plane of the sample, depending on the orientation of the Janus vector $\hat{\mathbf{s}}$ (normal to the metal-dielectric interface) (Supplemental Material Movie S1 [25]). Real-time trajectories of selected particles are grouped in Fig. 1(d). The dielectric hemispheres [Fig. 1(d), III and VII] lead when movement is parallel to, and the metal hemisphere [Fig. 1(d), I and V] when it is perpendicular to, the macroscopic director. For particles moving at other angles the Janus vector $\hat{\mathbf{s}}$ interpolates smoothly between

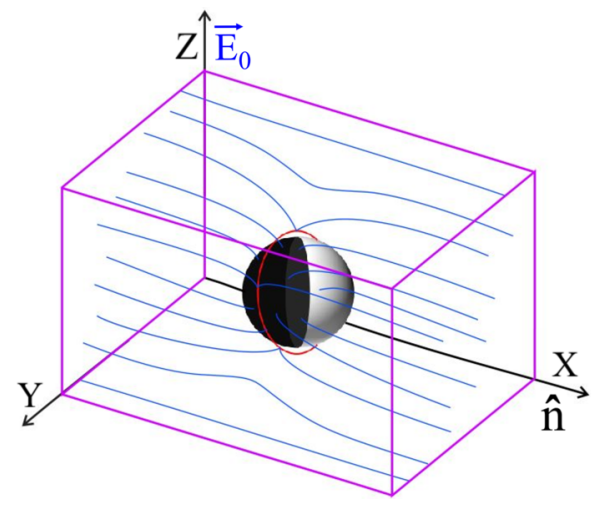

FIG. 2. Quadrupolar director field surrounding a Janus particle. The applied electric field $\mathbf{E}$ is transverse to the director. The red circle denotes the Saturn-ring defect. The black hemisphere represents metal.

these two extremes [Fig. 1(d), II, IV, VI, and VIII], and the particles can thus move in any direction in the plane of the sample as shown in Supplemental Material Fig. S2B [25].

To understand the motility of a Janus particle in an AC electric field we calculate the electrostatic force density induced by the field in the region around the particle. This force density drives fluid flow over the surface of the particle in such a way as to turn the particle into a swimmer, as sketched in Fig. 3.

We consider a single spherical Janus particle of radius $a$ in an NLC. The particle's surface consists of a conducting and a dielectric hemisphere, both of which are taken to impose identical uniform homeotropic surface anchoring on the ambient nematic, so as to produce an elastic quadrupolar distortion in the director field, that is, a radial hedgehog compensated by a Saturn-ring defect. An electric field $\mathbf{E}_{\mathbf{0}}$ along $z$ is externally imposed on the system as shown in Fig. 2. The mean macroscopic director field lies parallel to the $x$ axis and local deviations from this mean direction are described by an angle $\theta$, positive for counterclockwise rotation. We consider a system with conductivities $\sigma_{\|}, \sigma_{\perp}$ and dielectric constants $\epsilon_{\|}, \epsilon_{\perp}$, for electric fields parallel (\|) and perpendicular $(\perp)$ to the director $\hat{\mathbf{n}}$ and define the anisotropies $\Delta \sigma=\sigma_{\|}-\sigma_{\perp}$ and $\Delta \epsilon=\epsilon_{\|}-\epsilon_{\perp}$. Our strategy, generalizing [24], is to use charge conservation and Gauss's law to obtain the total electric field $\mathbf{E}$ and the charge density $\rho$ and thus the electrostatic force density, $\mathcal{F}(\mathbf{r})=\rho(\mathbf{r}) \mathbf{E}(\mathbf{r})$, separately for the case of a dielectric and a conducting sphere and to combine these results to infer the character of the induced flow around the Janus sphere.

For our particles of radius $a \simeq 1.5 \mu \mathrm{m}$ moving at speed $v \simeq 10 \mu \mathrm{m} / \mathrm{s}$ through a liquid crystal of mass density $\rho \simeq$ $10^{3} \mathrm{~kg} / \mathrm{m}^{3}$, shear viscosity $\eta \simeq 0.02 \mathrm{~Pa} \cdot \mathrm{s}$, Frank elastic constants $K \simeq 20 \mathrm{pN}$ (values quoted by the supplier Merck $\mathrm{KGaA}$ for our sample of MLC-6608), Reynolds number $\mathrm{Re}=$ $\rho v a / \eta \simeq 10^{-7}$, and Ericksen number $\mathrm{Er}=\eta v a / K \simeq 10^{-2}$. We can therefore ignore the effect of fluid inertia and we can take the director configuration around the particles to be negligibly influenced by fluid flow. As in [24], we work at zero Peclet number, i.e., take the charge currents to be purely ohmic and not advected by fluid flow. For simplicity 
we work at low frequencies so that time dependence can be neglected in the induction equation and thus the electric field $\mathbf{E}=-\nabla \Psi$, where $\Psi$ is a potential. We work to first order in the anisotropies $\Delta \sigma$ and $\Delta \epsilon$. We begin by evaluating the potential for a conducting or dielectric particle in the absence of anisotropy, for which the electrostatic boundary conditions imply

$$
\begin{gathered}
\Psi_{0} \equiv-E_{0} z+\lambda E_{0} \Phi \equiv-E_{0} z+\lambda \frac{E_{0} a^{3} z}{r^{3}}, \\
\lambda= \begin{cases}1, & \text { conductor, } \\
\frac{\epsilon_{r}-1}{\epsilon_{r}+2}<1, & \text { dielectric, }\end{cases}
\end{gathered}
$$

where $\epsilon_{r}$ is the dielectric constant.

To calculate the induced charge density in the nematic due to anchoring, we impose steady-state charge conservation $\nabla \cdot \mathbf{J}=0$ for a current $\mathbf{J}=\boldsymbol{\sigma} \cdot \mathbf{E}=\left(\sigma_{\perp} \mathbf{I}+\Delta \sigma \hat{\mathbf{n}} \hat{\mathbf{n}}\right) \cdot(-\nabla \Psi)$, where $\mathbf{I}$ is the unit tensor. This implies

$$
\left(\sigma_{\perp} \mathbf{I}+\Delta \sigma \hat{\mathbf{n}} \hat{\mathbf{n}}\right): \nabla(\nabla \Psi)=-\Delta \sigma(\hat{\mathbf{n}} \nabla \cdot \hat{\mathbf{n}}+\hat{\mathbf{n}} \cdot \nabla \hat{\mathbf{n}}) \cdot \nabla \Psi,
$$

where the colon (:) denotes contraction with both indices of $\nabla(\nabla \Psi)$. For small deviations $\delta \hat{\mathbf{n}}$ about a mean alignment $\hat{\mathbf{n}}_{0}$ and corresponding deviations $\delta \mathbf{E}$ from a field $\mathbf{E}=-\nabla \Psi_{0}$ imposed from the boundaries, writing $\delta \mathbf{E}=-\nabla \delta \Psi$ in terms of a potential $\delta \Psi$, (2) becomes

$$
\begin{aligned}
\left(\sigma_{\perp} \nabla_{\perp}^{2}+\sigma_{\|} \nabla_{\|}^{2}\right) \Psi= & -\Delta \sigma\left[\left(\hat{\mathbf{n}}_{0} \nabla \cdot \delta \hat{\mathbf{n}}+\hat{\mathbf{n}}_{0} \cdot \nabla \delta \hat{\mathbf{n}}\right) \cdot \nabla \Psi_{0}\right. \\
& \left.+\left(\hat{\mathbf{n}}_{0} \delta \hat{\mathbf{n}}+\delta \hat{\mathbf{n}} \hat{\mathbf{n}}_{0}\right): \nabla\left(\nabla \Psi_{0}\right)\right],
\end{aligned}
$$

where $\|$ and $\perp$ denote components along and transverse to $\hat{\mathbf{n}}_{0}$ and $\Psi=\delta \Psi+\Psi_{0}$. Next, Gauss's law $\rho=\nabla \cdot \mathbf{D}=\epsilon_{0} \nabla$. $(\boldsymbol{\epsilon E})$ reads, in the same linearized approximation,

$$
\begin{gathered}
\rho=-\epsilon_{0}\left(\epsilon_{\perp} \nabla_{\perp}^{2}+\epsilon_{\|} \nabla_{\|}^{2}\right) \Psi-\epsilon_{0} \Delta \epsilon\left[\left(\hat{\mathbf{n}}_{0} \nabla \cdot \delta \hat{\mathbf{n}}+\hat{\mathbf{n}}_{0}\right.\right. \\
\left.\cdot \nabla \delta \hat{\mathbf{n}}) \cdot \nabla \Psi_{0}+\left(\hat{\mathbf{n}}_{0} \delta \hat{\mathbf{n}}+\delta \hat{\mathbf{n}} \hat{\mathbf{n}}_{0}\right): \nabla\left(\nabla \Psi_{0}\right)\right] .
\end{gathered}
$$

Solving (3) for $\Psi$ allows us to write the force density

$$
\begin{aligned}
\mathcal{F}= & \rho \mathbf{E} \simeq \epsilon_{0}\left(-\Delta \epsilon+\Delta \sigma G_{\epsilon}^{-1} G_{\sigma}\right)\left[\left(\hat{\mathbf{n}}_{0} \nabla \cdot \delta \hat{\mathbf{n}} \hat{\mathbf{n}}_{0} \cdot \nabla \delta \hat{\mathbf{n}}\right)\right. \\
& \left.\cdot \nabla \Psi_{0}+\left(\hat{\mathbf{n}}_{0} \delta \hat{\mathbf{n}}+\delta \hat{\mathbf{n}} \hat{\mathbf{n}}_{0}\right): \nabla\left(\nabla \Psi_{0}\right)\right] \mathbf{E}_{0},
\end{aligned}
$$

where we have defined the Green's functions

$$
G_{\sigma}=\left(\sigma_{\perp} \nabla_{\perp}^{2}+\sigma_{\|} \nabla_{\|}^{2}\right)^{-1}
$$

and

$$
G_{\epsilon}=\left(\epsilon_{\perp} \nabla_{\perp}^{2}+\epsilon_{\|} \nabla_{\|}^{2}\right)^{-1} .
$$

At the lowest order in $\delta \hat{\mathbf{n}}$ the charge density is driven by the externally imposed electric field $\mathbf{E}_{0}$. We show below that the second term (which we call $\mathcal{F}_{I I}$ ) in brackets on the right-hand side of (5) contributes only a higher multipole to the force density. The force density thus takes the form

$$
\begin{aligned}
\mathcal{F} \simeq & \epsilon_{0}\left(-\Delta \epsilon+\Delta \sigma G_{\epsilon}^{-1} G_{\sigma}\right)\left[\left(\hat{\mathbf{n}}_{0} \nabla \cdot \delta \hat{\mathbf{n}}+\hat{\mathbf{n}}_{0}\right.\right. \\
& \left.\cdot \nabla \delta \hat{\mathbf{n}}) \cdot \nabla \Psi_{0}\right] \mathbf{E}_{0} .
\end{aligned}
$$

It is useful to decompose the force density as $\mathcal{F}=\mathcal{F}_{0}+\mathcal{F}_{\lambda}$, where $\mathcal{F}_{0}$ is a contribution independent of whether the sphere is dielectric or conducting, while $\mathcal{F}_{\lambda}$ depends, through $\lambda$, on the electrical nature of the sphere,

$$
\begin{aligned}
\mathcal{F}_{\mathbf{0}} \simeq & -\epsilon_{0}\left(-\Delta \epsilon+\Delta \sigma G_{\epsilon}^{-1} G_{\sigma}\right)\left(\hat{\mathbf{n}}_{0} \nabla \cdot \delta \hat{\mathbf{n}}+\hat{\mathbf{n}}_{0}\right. \\
& \cdot \nabla \delta \hat{\mathbf{n}}) \cdot \hat{\mathbf{z}} E_{0}^{2} \hat{\mathbf{z}}
\end{aligned}
$$

and

$$
\begin{aligned}
\mathcal{F}_{\lambda} \simeq \epsilon_{0} \lambda\left(-\Delta \epsilon+\Delta \sigma G_{\epsilon}^{-1} G_{\sigma}\right)\left(\hat{\mathbf{n}}_{0} \nabla \cdot \delta \hat{\mathbf{n}}+\hat{\mathbf{n}}_{0}\right. \\
\cdot \nabla \delta \hat{\mathbf{n}}) \cdot \nabla \Phi E_{0}^{2} \hat{\mathbf{z}},
\end{aligned}
$$

where $\Phi$ is defined in (1) above, and $\mathcal{F}_{0}$ is the same for both dielectric and conducting surfaces, while the symmetrybreaking piece is $\mathcal{F}_{\lambda}$. From (9) and (10), we see that the effect is proportional to the square of the electric field and thus survives time averaging over a period. Also, from (10), the force dipole is larger for a conducting sphere than for a dielectric sphere by a factor of $1 / \lambda$. Therefore, for a Janus particle, the center of the force dipole shifts from the geometric center towards the conducting side, breaking the symmetry and hence rendering the particle motile in an applied AC electric field.

For Fig. 2, we consider a local Cartesian coordinate system and small-angle approximation, $\hat{\mathbf{n}}_{0} \simeq \hat{\mathbf{x}}$ and $\delta \hat{\mathbf{n}} \sim$ $(0, \theta(x, z))$. The second term in (5) reduces to $\mathcal{F}_{\boldsymbol{I I}} \simeq$ $2 \epsilon_{0}\left(-\Delta \epsilon+\Delta \sigma G_{\epsilon}^{-1} G_{\sigma}\right) \theta \partial_{x} \partial_{z} \Psi_{0} \mathbf{E}_{0}$, with signs $(-+-+)$ for positive $z$ and $(+-+-)$ for negative $z$, as one moves from positive $x$ towards negative $x$, and hence has only a higher multipole contribution. The force density of (8), on the other hand, reads

$$
\mathcal{F} \simeq \epsilon_{0}\left(-\Delta \epsilon+\Delta \sigma G_{\epsilon}^{-1} G_{\sigma}\right)\left(\frac{\partial \theta}{\partial z} \frac{\partial \Psi_{0}}{\partial x}+\frac{\partial \theta}{\partial x} \frac{\partial \Psi_{0}}{\partial z}\right) E_{0} \hat{\mathbf{z}}
$$

In Fig. 2 we see that the director curvatures in (11) are composed of bend concentrated just outside the Saturn ring $\left(\partial_{x} \theta\right)$ coupled to $\left(\partial_{z} \Psi_{0}\right)$ and splay on the particle surface $\left(\partial_{z} \theta\right)$ coupled to $\left(\partial_{x} \Psi_{0}\right)$. We see that the signs in the four quadrants are $\partial_{x} \theta(--++), \partial_{z} \Psi_{0}(----), \partial_{z} \theta(+--+)$, and $\partial_{x} \Psi_{0}(-+-+)$. From (6) and (7) we see that although $G_{\epsilon}^{-1} G_{\sigma}$ has a nonlocal piece decaying as $1 / r^{3}$, it is formally a positive operator if examined in Fourier space. For our system $\Delta \epsilon<0$ and $\Delta \sigma>0$. Therefore, the splay contribution produces a force dipole of the contractile or puller type, while the bend produces a force dipole of the extensile or pusher type with respect to the electric-field axis.

Given that the electric field, via (11), results in a force density along $z$, we can understand trajectories III and VII [see Fig. 1(d)] by asking how the Janus character breaks symmetry in the $x z$ plane. In this plane the splay contribution is greater than the bend, as it is present over a larger part of the particle surface. Shifting the force dipole towards the metallic side yields a puller-type force dipole resulting in motility with the dielectric face forward as shown in Fig. 3(a). To test whether this idea makes sense let us apply it to the case of trajectories I and V in Fig. 1(d). Here the breaking of symmetry in the $y z$ plane is of relevance. Bend all along the Saturn ring is in play, which gives a pusher force dipole. Shifting this towards the metal face results in motility with the conductor face forward as shown in Fig. 3(b). The consistency of our explanation for the cases of the Janus axis parallel and perpendicular to 
(a)

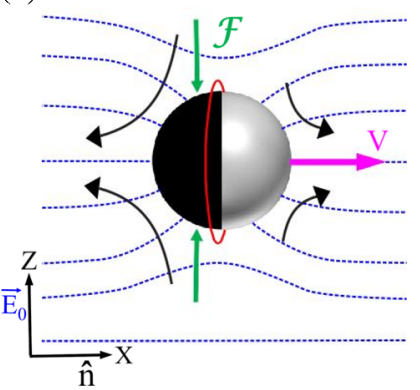

(b)

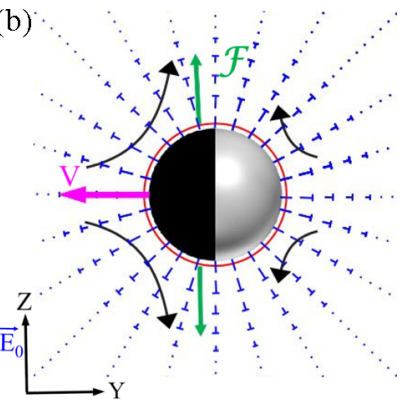

FIG. 3. Liquid-crystal-enabled electro-osmotic flows around a quadrupolar Janus particle in two orthogonal planes parallel to the electric field. Red circles represent Saturn-ring defects. The black hemisphere represents metal. The large curved arrows around the metal hemisphere indicate stronger flows. The off-center force dipoles $\mathcal{F}$ (shown in green) are (a) pullers and (b) pushers. The propulsion direction of the particles is indicated by a pink arrow for (a) trajectory III/VII and (b) trajectory I/V.

the macroscopic nematic alignment is reassuring. For other trajectories motility is due to a combination of both the effects stated above and therefore interpolates in direction.
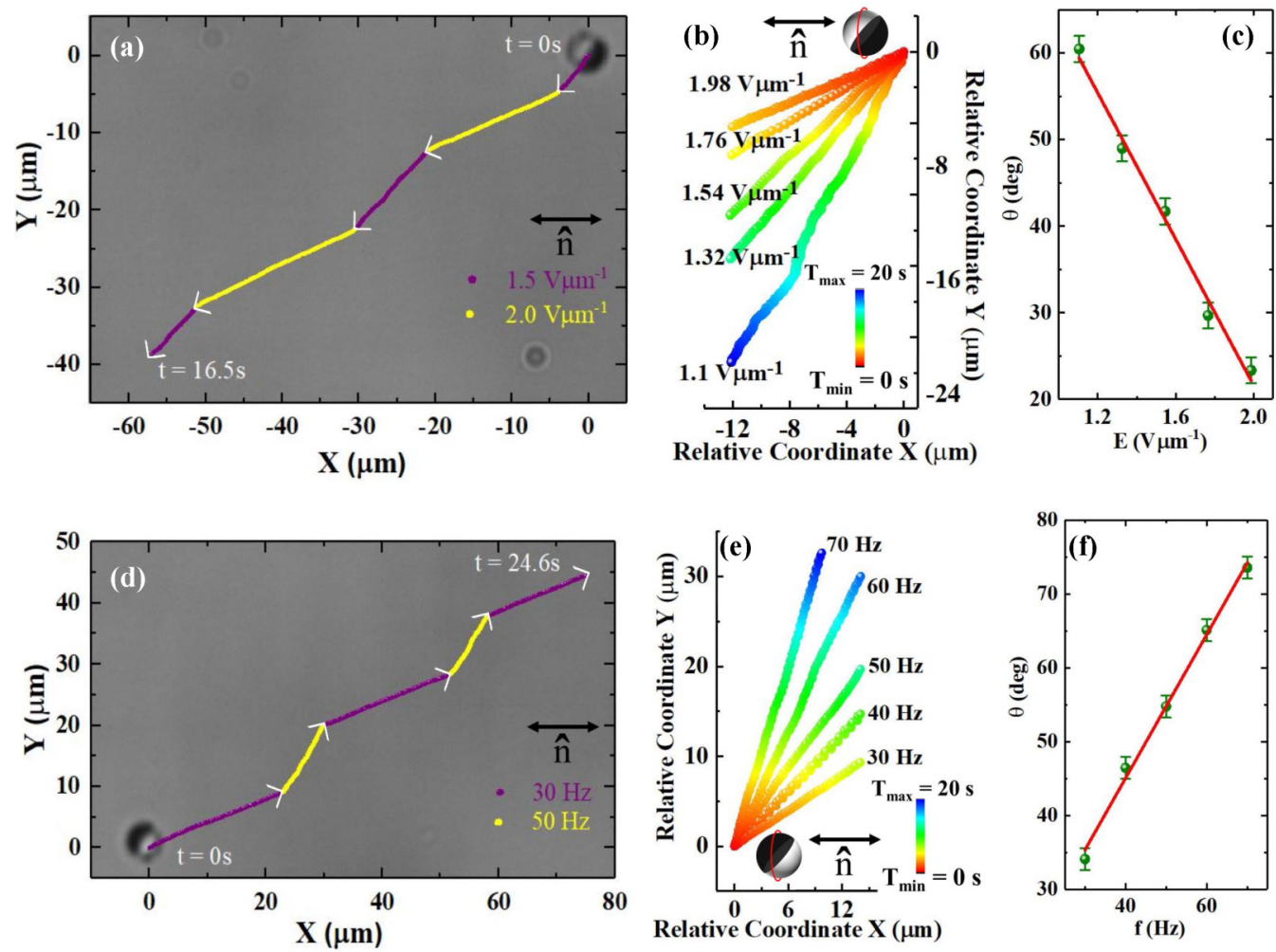

FIG. 4. (a) Change in the direction of motion of a quadrupolar Janus particle upon altering the field amplitude recursively between

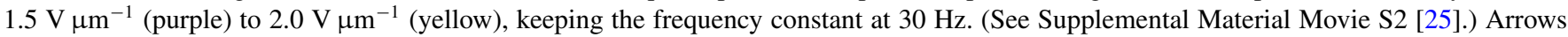
indicate the direction of motion. (b) Time-coded trajectories of a particle at different field amplitudes and a fixed frequency (30 Hz). (c) The angle between the trajectory and the director $(\theta)$ decreases linearly with the field at a slope of $-43.2^{\circ} \pm 2.3^{\circ} \mu \mathrm{m} \mathrm{V} \mathrm{V}^{-1}$. (d) Change in the direction of motion of a particle upon altering the frequency recursively between $30 \mathrm{~Hz}$ (purple) and $50 \mathrm{~Hz}$ (yellow), keeping the field amplitude constant at $1.5 \mathrm{~V} \mathrm{\mu m}^{-1}$. (See Supplemental Material Movie S3 [25].) (e) Time-coded trajectories of a particle at different frequencies and a

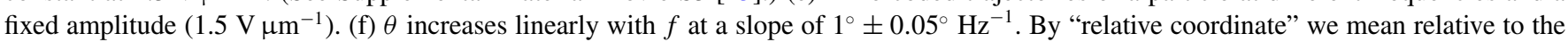
starting point of each trajectory. Error bars represent the standard deviation of the mean value. Cell thickness: $5.2 \mu \mathrm{m}$. 

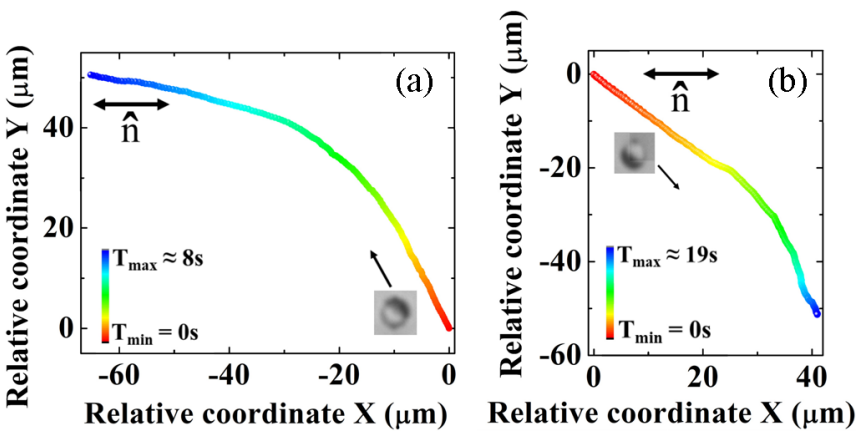

FIG. 5. (a) A particle with $\varphi=135^{\circ}$ is piloted to a predetermined place by changing the amplitude of the field (see Supplemental Material Movie S4 [25]). The field amplitude is increased in a continuous manner from 1.2 to $2.0 \mathrm{~V} \mu \mathrm{m}^{-1}$, keeping the frequency fixed at $30 \mathrm{~Hz}$. (b) A particle with $\varphi=315^{\circ}$ is transported to a predetermined place by increasing the frequency in a continuous manner from 20 to $90 \mathrm{~Hz}$, keeping the amplitude fixed at $1.6 \mathrm{~V} \mathrm{\mu m}^{-1}$ (see Supplemental Material Movie S5 [25]). Arrows near the particles show the initial direction of motion. Cell thickness: $5.2 \mu \mathrm{m}$.

of the field at a fixed frequency. Similarly, a particle with $\varphi=315^{\circ}$ is guided to a specified destination by increasing the frequency while keeping the amplitude fixed [see Fig. 5(b)]. In both cases the direction of motion changes continuously while the orientation of the Janus vector $\hat{\mathbf{s}}$ remains unchanged (see Supplemental Material Movies S4 and S5 [25]).

To understand the navigation of the Janus quadrupolar particles, we measured the field dependence of the velocity (see Supplemental Material Fig. S3 [25]). The motion of the particles shown in Figs. 4(a) and 4(d) has two velocity components, namely, $V_{x}$ and $V_{y}$, along the $x$ and $y$ directions, respectively, both of which are proportional to $E^{2}$, but the slope of $V_{x}$ is larger than that of $V_{y}$ [see Fig. 6(a)].

When the field is increased, the relative enhancements are unequal, i.e., $\Delta V_{x}$ is larger than $\Delta V_{y}$, and consequently, $\theta=$ $\tan ^{-1}\left(\Delta V_{y} / \Delta V_{x}\right)$ decreases. The velocity components also
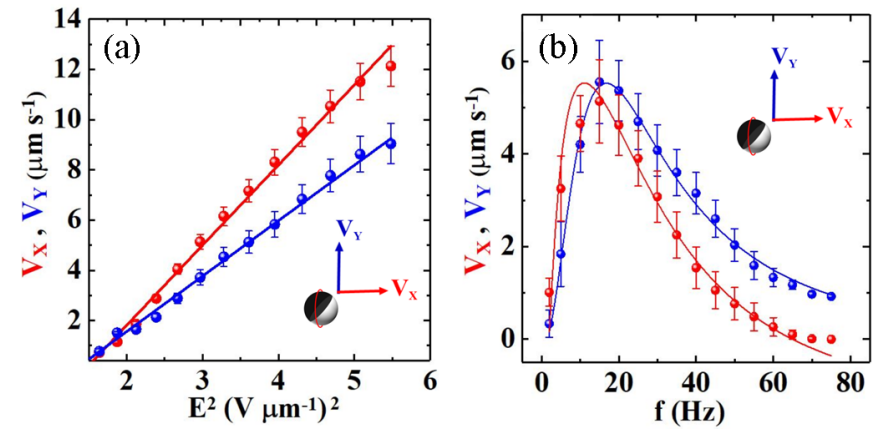

FIG. 6. (a) Dependence of the velocity components $V_{x}$ and $V_{y}$ of a Janus particle [trajectory II; Fig. 1(d)] on the electric-field amplitude $E$ at $f=30 \mathrm{~Hz}$. Solid lines show least-squares fits to $V \propto E^{2}$. The slopes of $V_{x}$ and $V_{y}$ are 3.2 and $2.2 \mu \mathrm{m}^{3} \mathrm{~V}^{-2} \mathrm{~s}^{-1}$, respectively. (b) Frequency-dependent $\left(E=1.54 \mathrm{~V} \mathrm{\mu m}^{-1}\right)$ velocity components $V_{x}$ and $V_{y}$. Red and blue lines show the theoretical fit to Eq. (2). Fit parameters are $\tau_{e}=0.25 \mathrm{~s}, \tau_{p}=0.032 \mathrm{~s}$ for $V_{x}$ and $\tau_{e}=0.08 \mathrm{~s}$, $\tau_{p}=0.04 \mathrm{~s}$ for $V_{y}$. Error bars represent the standard deviation of the mean value. Cell thickness: $5.2 \mu \mathrm{m}$. depend strongly on the frequency $(f)$ of the field [see Fig. 6(b)]. Both the components show the frequency dependence given by $[11,23]$

$$
V_{i}(\omega)=V_{i}^{o} \frac{\omega^{2} \tau_{e}^{2}}{\left(1+\omega^{2} \tau_{p}^{2}\right)\left(1+\omega^{2} \tau_{e}^{2}\right)},
$$

where $i=x, y, \omega=2 \pi f$ is the angular frequency of the applied field, and $\tau_{e}$ and $\tau_{p}$ are the characteristic electrode and particle charging time, respectively. Both the velocity components increase as $f^{2}$ in the low-frequency regime but decrease as $f^{-2}$ at higher frequencies because the ions cannot follow the rapidly changing field. For frequencies above $15 \mathrm{~Hz}$ both $V_{x}$ and $V_{y}$ are proportional to $1 / f^{2}$ but the coefficient of the decrease in $V_{x}$ is larger than that for $V_{y}$, resulting in an increase in the angle $\theta$ when $f$ is increased.

Two further remarks are in order. First, for induced-charge electro-osmosis in an isotropic medium the flows are always of the puller type with respect to the electric-field axis [33], ruling out directional control of the type we discuss. Second, we expect that hydrodynamic torques arising from the coupling between the squirmer flow field and the anisotropic viscosity of the NLC [34] cannot operate here, as the orientation of the force dipole driving our swimming particles is determined by the direction of the imposed electric field. This rules out spontaneous change in the direction of motion of particles due to such torques at a fixed field and frequency.

We have shown that metal-dielectric Janus particles in an NLC film subjected to a perpendicular AC electric field behave like steerable active particles whose direction of motion can be dictated purely by varying the field amplitude and frequency. The underlying mechanism involves the contrasting electrostatic boundary conditions on the two Janus faces of the particles, the dielectric anisotropy of the nematic, and anchoring on the particle surfaces. We show that the time-averaged electrostatic force density produced around the Janus particle by the AC field is that of a force dipole whose center is shifted towards the conducting face, causing the particle to swim in the plane transverse to the field, with the dielectric (metal) face forward for a particle axis parallel (perpendicular) to the nematic director and interpolating smoothly for intermediate orientations. Studies on motility at higher concentrations, as well as collective dynamics as for diffusiophoretic active colloids [35,36], are natural experimental and theoretical challenges. Our study has focused on spherical particles in nematics with perpendicular surface anchoring and linear macroscopic alignment, but we have preliminary results for particles with planar surface anchoring, as well as for a racetrack director configuration. The abundance of new particles with controlled shapes, surface anchoring [37], and genus [38] now available and their extraordinary topological [39] and dynamical properties [40,41] promise a wide range of as yet unexplored physical effects and their applications.

S.D. thanks Steve Granick for hosting his visit to IBS, UNIST, which resulted in very useful discussions. S.D. also acknowledges Myeonggon Park and Joonwoo Jeong of UNIST for various discussions. We thank O. D. Lavrentovich, I. Muševič, S. Bhattacharya, and P. Anantha Lakshmi for useful discussions. We also thank K. V. Raman for help in 
preparing Janus particles. This work was supported by the DST, Government of India (DST/SJF/PSA-02/2014-2015). S.D. acknowledges a Swarnajayanti Fellowship and D.K.S. an
INSPIRE Fellowship from the DST. S.R. was supported by a J. C. Bose Fellowship from the SERB, India, and by the Tata Education and Development Trust.
[1] A. Ramos, Electrokinetics and Electrohydrodynamics in Microsystems (Spinger, Berlin, 2011).

[2] H. Morgan and N. G. Green, AC Electrokinetics: Colloids and Nanoparticles (Research Studies Press Ltd., 2003).

[3] S. V. Dorp, U. F. Keyser, N. H. Dekker, C. Dekker, and S. G. Lemay, Nat. Phys. 5, 347 (2009).

[4] J. Yan, M. Han, J. Zhang, C. Xu, E. Luijten, and S. Granick, Nat. Mater. 15, 1095 (2016).

[5] A. F. Demirörs, F. Eichenseher, M. J. Loessner, and A. R. Studart, Nat. Commun. 8, 1872 (2017).

[6] A. Terray, J. Oakey, and D. W. M. Marr, Science 296, 1841 (2002).

[7] B. Comiskey, J. D. Albert, H. Yoshizawa, and J. Jacobson, Nature 394, 253 (1998).

[8] R. C. Hayward, D. A. Saville, and I. A. Aksay, Nature 404, 56 (2000).

[9] V. A. Murtsovkin, Colloid J. 58, 341 (1996).

[10] T. M. Squires and S. R. Quake, Rev. Mod. Phys. 77, 977 (2005).

[11] M. Z. Bazant, M. S. Kilic, B. D. Storey, and A. Ajdari, Adv. Colloid Interface Sci. 152, 48 (2009).

[12] T. M. Squires and M. Z. Bazant, J. Fluid Mech. 509, 217 (2004).

[13] M. Z. Bazant and T. M. Squires, Phys. Rev. Lett. 92, 066101 (2004).

[14] S. Gangwal, O. J. Cayre, M. Z. Bazant, and O. D. Velev, Phys. Rev. Lett. 100, 058302 (2008).

[15] F. Ma, X. Yang, H. Zhao, and N. Wu, Phys. Rev. Lett. 115, 208302 (2015).

[16] S. Ramaswamy, R. Nityananda, V. A. Raghunathan, and J. Prost, Mol. Cryst. Liq. Cryst. 288, 175 (1996).

[17] Y. Gu and N. L. Abbott, Phys. Rev. Lett. 85, 4719 (2000).

[18] P. Poulin, H. Stark, T. C. Lubensky, and D. A. Weitz, Science 275, 1770 (1997).

[19] H. Stark, Phys. Rep. 351, 387 (2001).

[20] O. D. Lavrentovich, Curr. Opin. Colloid Interface Sci. 21, 97 (2016).

[21] O. D. Lavrentovich, Soft Matter 10, 1264 (2014).

[22] I. Lazo and O. D. Lavrentovich, Phil. Trans. R Soc. A 371, 2012255 (2013).
[23] O. D. Lavrentovich, I. Lazo, and O. P. Pishnyak, Nature 467, 947 (2010).

[24] I. Lazo, C. Peng, J. Xiang, S. V. Shiyanovskii, and O. D. Lavrentovich, Nat. Commun. 5, 5033 (2014).

[25] See Supplemental Material at http://link.aps.org/supplemental/ 10.1103/PhysRevResearch.2.032009 for movies, details of the experiments, and additional figures.

[26] R. Golestanian, arXiv:1909.03747.

[27] I. Muševič, M. Škarabot, U. Tkalec, M. Ravnik, and S. Žumer, Science 313, 954 (2006).

[28] J. Cleaver and W. C. K. Poon, J. Phys: Condens. Matter 16, S1901 (2004)

[29] T. A. Wood, J. S. Lintuvuori, A. B. Schofield, D. Marenduzzo, and W. C. K. Poon, Science 334, 79 (2011).

[30] O. P. Pishnyak, S. Tang, J. R. Kelly, S. V. Shiyanovskii, and O. D. Lavrentovich, Phys. Rev. Lett. 99, 127802 (2007).

[31] M. Conradi, M. Ravnik, M. Bele, M. Zorko, S. Žumer, and I. Muševič, Soft Matter 5, 3905 (2009).

[32] R. Mangal, K. Nayani, Y. K. Kim, E. Bukusoglu, U. M. Córdova-Figueroa, and N. L. Abbott, Langmuir 33, 10917 (2017).

[33] T. M. Squires and M. Bazant, J. Fluid Mech. 560, 65 (2006).

[34] J. S. Lintuvuori, A. Würger, and K. Stratford, Phys. Rev. Lett. 119, 068001 (2017).

[35] S. Saha, S. Ramaswamy, and R. Golestanian, New J. Phys. 21, 063006 (2019).

[36] S. Saha, R. Golestanian, and S. Ramaswamy, Phys. Rev. E 89 , 062316 (2014).

[37] B. Senyuk, O. Puls, O. M. Tovkach, S. B. Chernyshuk, and I. I. Smalyukh, Nat. Commun. 7, 10659 (2016).

[38] B. Senyuk, Q. Liu, S. He, R. D. Kamien, T. C. Lubensky, and I. I. Smalyukh, Nature 493, 200 (2013).

[39] Y. Yuan, Q. Liu, B. Senyuk, and I. I. Smalyukh, Nature 570, 214 (2019).

[40] S. Hernàndez-Navarro, P. Tierno, J. A. Farrera, J. Ignés-Mullol, and F. Sagués, Angew. Chem. Int. Ed. 53, 10696 (2014).

[41] A. V. Straube, J. M. Pagés, P. Tierno, J. Ignés-Mullol, and F. Sagués, Phys. Rev. Res. 1, 022008(R) (2019). 